\title{
Multiple DRO CRF escape avoidance: Contributions of shock duration and response requirements on avoidance
}

\author{
RICHARD PISACRETA \\ Ferris State College, Big Rapids, Michigan 49307
}

\begin{abstract}
Three rats were trained to escape electric shock by satisfying a continuous (CRF) and a differential reinforcement of other behavior (DRO) escape schedule. These escape schedules, accompanied by visual and auditory cues, were randomly alternated at 5-min intervals in a multipleschedule format. Each escape schedule was linked with a 20-sec avoidance component (R-S=20 sec), in which responses prevented the occurrence of escape periods. Later, a differential reinforcement of low rate (DRL) escape schedule was paired with the DRO escape schedule. In general, all subjects produced the high (CRF) and low (DRO and DRL) escape rates expected. During the shock-off intervals, two of the three rats maintained higher avoidance in the CRF escape components relative to the DRO escape components, although DRO escape involved longer escape periods. The differential escape rates and avoidance performances indicated reliable stimulus control by the various cues associated with the different escape avoidance schedules employed.
\end{abstract}

The free operant escape avoidance paradigm is frequently employed as a baseline schedule in much of the negative reinforcement literature. The procedure provides two components. During the escape component, aversive stimuli, typically electric shocks, are presented to the subject. The rat is the most frequently used animal. When the rat emits an escape response (e.g., a leverpress), the aversive stimuli are removed and a shock-free avoidance component begins. Additional responding during the shock-off periods (avoidance responses) delay the return of the next escape period.

Several researchers, however, have pointed out that half the animals fail to acquire or maintain superior avoidance levels (Bolles, 1971; Herrnstein, 1969; Meyer, Cho, \& Weseman, 1960; Pisacreta, 1980; Weismann, 1962).

Several experiments have been conducted to isolate factors that contribute to the establishment and maintenance of avoidance behavior. The aversiveness of a negative reinforcer is specified in terms of the intensity, frequency and duration of contact. Goldstein (1960), Herrnstein and Hineline (1966), McAllister and McAllister (1962), and Sidman (1962) have demonstrated that shock intensity and frequency influence the level of avoidance maintained. Leander (1973) and Riess (1970) demonstrated that the avoidance rates directly varied with the intensity and duration of the shocks presented during the escape periods. Pisacreta $(1980,1981)$ showed that various intermittent escape schedules improved avoidance performance originally

I wish to thank Nancy Myers for typing the final draft of the paper. Reprints may be obtained from Richard Pisacreta, Department of Psychology, Ferris State College, Big Rapids, Michigan 49307. established with a continuous (CRF) escape schedule. Intermittent escape schedules used included: differential reinforcement of low rate, (DRL), variable interval (VI), fixed interval (FI), and a tandem FI/differential reinforcement of high rate schedule, $(\tan$ FI/DRH). These schedules improved avoidance when employed alone and maintained different levels of avoidance when presented in pairs in a multiple-schedule format. A question remains as to why intermittent escape schedules improve avoidance. One possibility rests on the fact that all intermittent escape schedules involve longer contact with shock than CRF escape. CRF escape typically produces escape latencies of less than 500 msec (Dinsmoor, 1968; Pisacreta, 1980, 1981). An alternate position is that intermittent escape schedules reinforce particular behaviors during the shock-on periods that would facilitate avoidance if these behaviors were also emitted during the shock-off periods. For example, DRL escape reinforces the formation of temporal discriminations. Temporal discriminations in the avoidance components improve avoidance (Anger, 1963). FI, VI, and tan FI/DRH escape schedules reinforce high response rates. If these rates continue to be emitted during the avoidance components, they will improve avoidance. Pisacreta $(1980,1981)$ showed that rats trained on these schedules emit shock-off (avoidance) rates two to four times higher than the rate necessary to avoid all programmed escape periods.

The present experiment was designed to determine whether intermittent escape schedules improve avoidance because they involve longer shock exposure periods or because they reinforce response patterns that aid avoidance. The experiment employed a DRO escape schedule and a CRF escape schedule. If shock duration improves avoidance, then DRO escape should maintain 
higher avoidance than CRF escape. DRO escape, however, reinforces all behavior except leverpressing. If this pattern emerges during the avoidance components, then DRO escape should produce lower avoidance than CRF escape, even though DRO escape involves longer shock duration.

During the last phase of the experiment, the DRO escape schedule was paired with a DRL escape schedule. Both schedules allow equal shock duration, but each reinforces a different escape response pattern (Pisacreta, 1981). Holding shock exposure equal, any difference in avoidance behavior can be attributed to the particular escape schedule response requirement.

\section{METHOD}

\section{Subjects}

The subjects were three experimentally naive male 90-120-dayold albino Wistar rats. All subjects were individually housed and allowed free access to food and water in their home cages throughout the experiment.

\section{Apparatus}

The experimental chamber was a standard operant conditioning chamber $30.5 \mathrm{~cm}$ long $\times 24.8 \mathrm{~cm}$ wide $\times 30.5 \mathrm{~cm}$ high. Its front and rear walls were metal; side walls and ceiling were clear Plexiglas. The floor of the chamber was composed of 16 stainless steel rods, $.952 \mathrm{~cm}$ in diameter, running parallel to the front and rear walls. A GE 18207.5 -W bulb, located $2.50 \mathrm{~cm}$ above the roof of the chamber, served as a houselight.

One Lehigh Valley lever protruded $2.22 \mathrm{~cm}$ from the front wall, $6.35 \mathrm{~cm}$ above the grid floor. The lever required a force of approximately $.25 \mathrm{~N}$ and an excursion of $2.0 \mathrm{~mm}$ to operate its microswitch. Tones were produced by a Mallory Sonoalert (Model SLG 28) set at an intensity of $70 \mathrm{~dB}$.

The chamber was housed in a sound-attenuated outer enclosure. The outer enclosure's ventilating fan and a white-noise generator provided masking noise during sessions. The shock source was a fixed-impedance shock generator that delivered $300 \mathrm{~V}$ ac through a $300-\mathrm{K}$ resistor placed in series with the subject. The shock was scrambled by a solid state device (Graefe $\&$ Pisacreta, 1977) and was delivered to the chamber grids, lever front and rear walls, and feces tray. The scrambler provided a 20 -msec shock pulse every $320 \mathrm{msec}$ of shock-on time. All experimental events were controlled by electromechanical equipment located in a separate room.

\section{Procedure}

Phase 1. CRF escape avoidance. Each rat was exposed to 101.5 -h sessions on a free operant escape avoidance schedule. The shock-shock interval was zero (continuous shock), and the response-shock interval was $20 \mathrm{sec}$ (S-S $=0$, R-S $=20 \mathrm{sec}$ ). The houselight and tone were on throughout each session. Shock intensity was the same for all subjects, $1 \mathrm{~mA}$, and remained unchanged for the entire experiment. When the shock came on, a single leverpress, the CRF escape response, terminated shock for $20 \mathrm{sec}$. Additional responding during the shock-off period, avoidance responses, delayed the onset of shock until $20 \mathrm{sec}$ had passed since the last response. This $\mathrm{R}-\mathrm{S}=20 \mathrm{sec}$ avoidance component remained unchanged for all subjects throughout the experiment. Sessions were always $1.5 \mathrm{~h}$ in length.

Phase 2. DRO 5-sec escape avoidance. For the next 10 sessions, each rat was required to satisfy a DRO 5 -sec escape schedule during the shock-on periods. During the escape periods, which were signaled by the houselight and tone pulsing at a $1-\mathrm{Hz}$ rate, shock terminated $5 \mathrm{sec}$ after its onset, provided that the rat did not leverpess. Each shock-on response reset the escape period to $5 \mathrm{sec}$.

Phase 3. For the next 10 sessions, each rat was trained on a multiple DRO 5-sec CRF escape avoidance schedule. With the houselight and tone on constantly, the CRF escape avoidance schedule of Phase 1 was in effect. In the alternate component, the DRO 5-sec escape avoidance schedule of Phase 2 was in effect. The houselight and tone pulsed at a $1-\mathrm{Hz}$ rate. Each component was $5 \mathrm{~min}$ in length and alternated randomly, with the restriction that no component could occur more than twice in a row. A component was not allowed to change during an escape period.

Phase 4. For the next 10 sessions, the requirement in the intermittent escape component was raised to DRO $10 \mathrm{sec}$.

Phase 5. The rats were placed on a multiple DRO 15-sec CRF escape avoidance schedule for 10 sessions.

Phases 6, 7, and 8. Phases 6-8 replicated the schedule conditions of Phases 4, 3, and 1, respectively.

Phase 9. For the 10 sessions of this phase, a DRL 5-sec escape avoidance schedule was in effect. During each escape component, the first response after 5 sec terminated shock and reinstated the avoidance component. Premature responses reset the DRL timer, thus lengthening the shock-on interval.

Phase 10. For the 10 sessions of the last phase, a multiple DRO 5-sec DRL 5-sec schedule was in effect.

\section{RESULTS}

\section{Escape}

Figure 1 shows the shock-on rates yielded by each rat during the last five sessions of each phase. Shock-on rates were derived by dividing the cumulative response frequency during the escape periods for each session by the shock-on time accumulated during those periods. The first panel shows that each rat produced a high escape rate during Phase 1. During Phase 2, the shock-on rate of each rat was reduced to near zero. During the next phase, each rat yielded a higher escape rate during the CRF escape components relative to the rates produced during the DRO 5-sec escape periods. Comparison of the CRF escape rates between Phases 1 and 3 indicates they were lower in Phase 3. This "induction" effect between the components was first demonstrated by Reynolds (1961), who employed positive reinforcers. Pisacreta (1981) provided a negative reinforcement

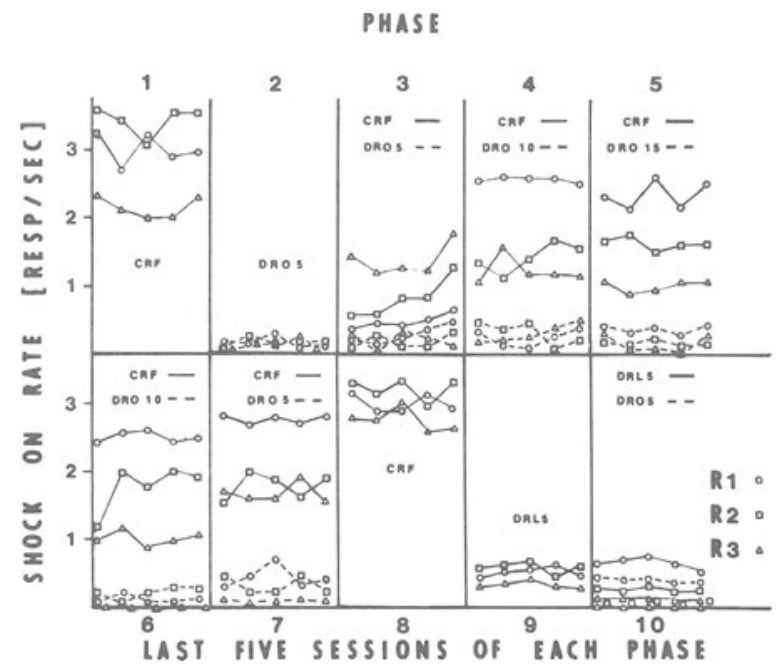

Figure 1. Shock-on rates produced during the last five sessions of each phase of the experiment. 
demonstration of the same phenomenon. This separation in the CRF and DRO escape rates was carried across the next four phases, Phases 4, 5, 6, and 7. The escape rates of Phase 8 replicated those produced in Phase 1 . The DRL 5-sec escape schedule of Phase 9 produced the low but sustained escape rates expected. Finally, during Phase 10, each rat produced a higher escape rate in the DRL 5-sec component relative to their DRO 5-sec escape rates.

\section{Average Shock-On Time}

Each rat produced the same short escape periods $(.5 \mathrm{sec})$ previously reported by Dinsmoor (1968) and Pisacreta (1980, 1981). Each rat yielded an average shock interval of $7 \mathrm{sec}$ during Phase 2. As expected, each rat maintained higher shock-on intervals during the DRO components relative to the $\mathrm{CRF}$ components during Phases 3-7 inclusive. With DRO 10-sec and DRO 15-sec escape, the average shock-on time was $12 \mathrm{sec}$ and $20 \mathrm{sec}$, respectively. During Phase 9, each rat produced shock-on intervals two to three times longer than the DRL 5-sec schedule required. Finally, during Phase 10 , each rat produced longer shock intervals during the DRL 5-sec escape component relative to those yielded in the DRO 5 -sec components.

\section{Avoidance Rates}

Figure 2 shows the shock-off avoidance rates produced during the last five session of each phase. Panel 1, Phase 1, shows that all the rats produced very stable shock-off rates during the components linked to CRF escape. Panel 2, Phase 2, shows that when DRO 5-sec escape was introduced, only Subject R1 maintained a high avoidance rate. During the five phases (Phases 3-7) that presented a multiple DRO/CRF escape avoidance schedule, two of the three rats reliably produced a higher avoidance rate in the CRF component relative to the avoidance rates maintained during the DRO escape components. Consistently across phases, R1

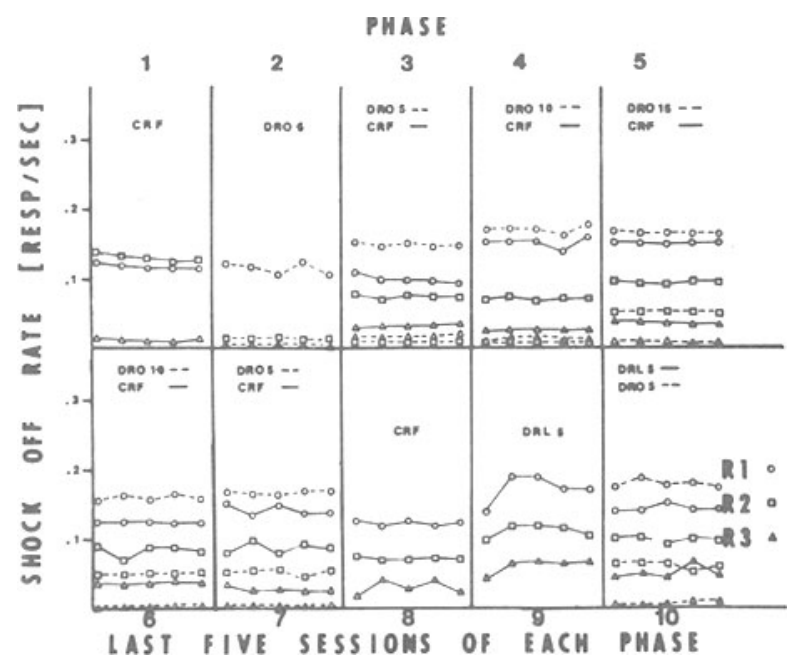

Figure 2. Shock off, avoidance rates produced during the last five sessions of each phase of the experiment.

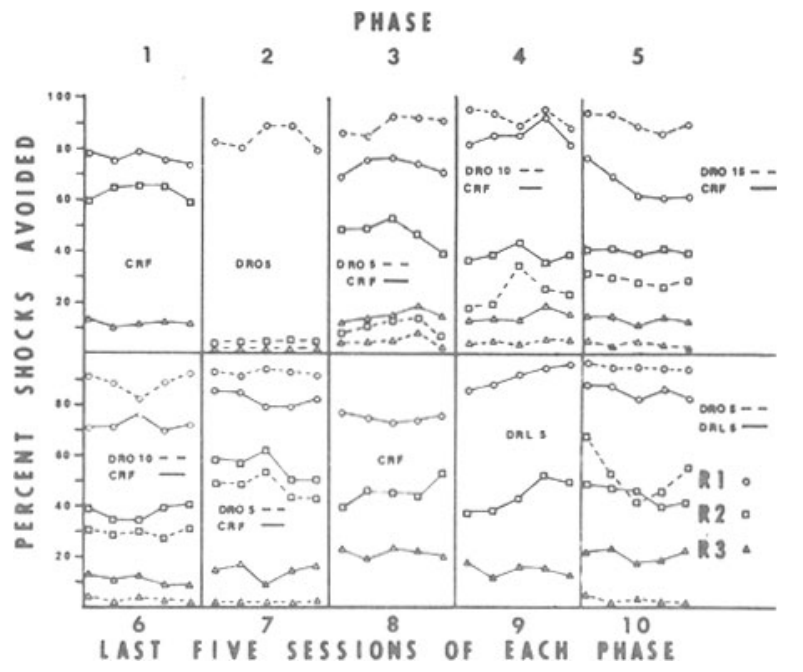

Figure 3. Percentage of shock-on periods avoided during the last five sessions of each phase of the experiment.

produced the highest avoidance rates. A comparison of Phase 9 with earlier phases shows that all rats produced a higher shock-off rate during sessions presenting DRL 5sec escape relative to the avoidance levels maintained by the alternative intermittent escape schedule (i.e., DRO escape). During Phase 10, two of the three rats produced higher avoidance rates in the DRL 5-sec escape component relative to the avoidance rates yielded during the DRO 5-sec escape components.

\section{Percent Shocks Avoided}

Figure 3 presents the percent of shock-on periods that were avoided during the last five sessions of each phase. Each time a rat escaped shock, the escape response also produced a 20 -sec shock-off period, the R-S interval.

In order to separate the shock-off time produced by avoidance responses from the shock-off time produced as a consequence of escape, the R-S interval value, $20 \mathrm{sec}$, was multiplied by the number of shocks and the product was subtracted from the total shock-off time recorded for the session. The result is the amount of shock-off time produced by shock-off (avoidance) responses. By dividing this shock-off time by the R-S interval, we obtain the number of potential shock-on periods that could have occurred but were avoided. By summing the number of actual shock-on periods received with the number of potential shock-on periods avoided, we account for all possible shock-on periods for the session, multiplying the result by 100 in order to convert it to a percent. Panel 1, Phase 1, shows that R1, R2, and R3 established high, medium, and low levels of avoidance, respectively. Rats typically yield various levels of avoidance (Bolles, 1971; Pisacreta, 1981). The introduction of DRO 5-sec escape in Phase 2 virtually eliminated avoidance behavior in R2 and R3. During Phases 3.7 inclusive, R2 and R3 avoided more programmed shock periods during the CRF escape components relative to the DRO escape components. That is, 
these rats avoided more shocks during the component that involved shorter shock-on periods but reinforced leverpresses. R1 reliably avoided more shocks during the DRO escape components. Pairing DRO escape with CRF escape improved the overall avoidance performance of $\mathrm{R} 2$ relative to Phase 2, in which DRO escape was presented alone. Furthermore, R2's avoidance improved during Phase 8 when DRF escape was presented alone. DRL 5-sec escape (Phase 9) did not disrupt avoidance for R2 and R3 as DRO 5-sec escape did in Phase 2.

The average shock-on times were twice as long during the DRL 5-sec escape periods relative to the DRO 5-sec escape periods. Figure 3 shows that R1 and R2 avoided more shocks during the DRO escape component. Reintroduction of the DRO escape schedule again eliminated avoidance in R3 during those components. A comparison of Figures 2 and 3 shows a strong correlation between shock-off rates and percent shocks avoided, for all subjects.

\section{DISCUSSION}

The present results are similar to those reported by Pisacreta (1980, 1981). They demonstrate that a multiple schedule employing a negative reinforcer in conjunction with different escape schedules is a viable research procedure. Similar to the appetitive literature, interactions between the two multiple-schedule components were observed. The escape rates in the CRF escape components of Phase 1 were high. The escape rates in the CRF escape components were lower when they were paired with the DRO escape components compared to when they were employed alone. Similar to the data presented by Pisacreta (1981), the present study also represents a negative reinforcement analogue to the appetitive literature (Reynolds, 1961).

Several researchers (e.g., Bolles, 1971; D’Amato \& Schiff, 1964; Seligman, 1970; Weismann, 1962) have reported that many rats fail to acquire satisfactory avoidance levels. The present results indicate that intermittent escape schedules influence avoidance levels initially established with CRF escape (see Pisacreta, 1980, 1981). Also similar to Pisacreta's results, the present study showed that intermittent escape schedules virtually eliminate the "warm-up effects" that several researchers have reported (Foree \& Lolordo, 1970; Hoffman, Fleshler, \& Chorny, 1961).

The data also indicate that rats are very sensitive to escape schedule changes. Several sessions are not required for stability to emerge. Furthermore, escape schedule manipulations produce stable changes in the first session in which they are introduced.

The present study indicates that three factors may contribute to the ability of intermittent escape schedules to influence avoidance performance. They are: the baseline avoidance level established with CRF escape, the shock duration requirement by the intermittent escape schedule, and the particular response pattern (e.g., high rate, low rate, no rate, temporal discrimination) that is required during the escape components. The present data suggest that a good avoider, $80 \%$ or better, may be more influenced by shock durations than by escape response requirements. In the present study, a medium avoider (40\%-60\%) and a poor avoider ( $20 \%$ or less) appear to be more influenced by the escape component response requirement. Specifically, Subject R1, a good avoider $(80 \%)$ increased his avoidance to 90\%-95\% during the phases that employed DRO escape. Rat R2, a medium avoider (60\%) during CRF escape sessions, yielded $5 \%$ avoidance when DRO escape was introduced. Subject R3, a poor avoider, yielded his best avoidance during sessions employing escape schedules that required an escape reponse, CRF and DRL relative to the poor avoidance DRO escape maintained. Interactions between the escape components may obscure these trends, as the avoidance results of Phase 10 may indicate. Nevertheless, this "interaction between escape schedule parameters and avoidance" position is also supported by the data published by Pisacreta $(1980,1981)$. Obviously, however, further work is required addressing the interdependence of aversive situations before a thorough, definitive, theory will emerge. Fixed-interval, fixed-ratio, variable-interval, and variable-ratio escape schedules should be employed alone and in multiple- and mixed-schedule formats. Other negative reinforcers, different species, and several operants should be parametrically explored.

\section{REFERENCES}

ANGER, D. The role of temporal discrimination in the reinforcement of Sidman avoidance behavior. Journal of the Experimental Analysis of Behavior, 1963, 6, 477-506.

Bolles, R. C. Species-specific defense reaction. In F. R. Brush (Ed.), Aversive conditioning and learning. New York: Academic Press, 1971.

D'Amato, M. R., \& Schiff, D. Long term discriminated avoidance training. Journal of Comparative and Physiological Psychology, 1964, 56, 123-126.

Dinsmoor, J. A. Escape from shock as a conditioning technique. In M. Jones, Miami Symposium on the Prediction of Behavior 1967: Aversive Stimulation. Coral Gables, Fla: University of Miami Press, 1968.

Foree, D. D., \& Lolordo, V. M. Signalled and unsignalled freeoperant avoidance in the pigeon. Journal of the Experimental Analysis of Behavior, 1970, 13, 283-290.

Goldste in, M. L. Acquired drive strength as a joint function of shock intensity and number of acquisition trials. Journal of Experimental Psychology, 1960, 60, 349-358.

Graefe, J., \& Pisacreta, R. An integrated circuit shock scrambler. Behavioral Research Methods \& Instrumentation, 1977, $9,499$.

HerRnstein, R. J. Method and theory in the study of avoidance. Psychological Review, 1969, 76, 49-69.

Herrnstein, R. J., \& Hineline, P. N. Negative reinforcement as shock frequency reduction. Journal of the Experimental Analysis of Behavior, 1966, 9, 421-430.

Hoffman, H. S., Fleshler, M., \& Chorney, H. Discriminated bar press avoidance. Journal of Experimental Analysis of Behavior, 1961, 4, 309-316.

LEANDER, D. Shock intensity and duration interaction on free operant avoidance behavior. Journal of the Experimental Analysis of Behavior, 1973, 19, 481-490.

Meyer, D. R., Cho, C., \& Weseman, A. F. On problems of conditioning discriminated lever-press avoidance responses. Psychological Review, 1960, 67, 224-228.

McAllister, W. R., \& McAllister, D. E. Post conditioning delay and intensity of shock as factors in the measurement of acquired fear. Journal of Experimental Psychology, 1962, 64, 110-116.

Pisacreta, R. Intermittent escape schedules and their effect on avoidance. Psychological Record, 1980, 30, 237-250.

Pisacreta, R. Multiple schedules of escape and their effects on avoidance. Psychological Record, 1981, 31, 573-587.

REYNolds, G. S. An analysis of interactions in a multiple schedule. Journal of the Experimental Analysis of Behavior, 1961, 4, 107-117.

RIEss, D. A shaping technique for producing rapid and reliable bar press avoidance. Journal of the Experimental Analysis of Behavior, 1970, 13, 279-282.

Seligman, M. E. P. On the generality of the laws of learning. Psychological Review, 1970, 77, 406-418.

Sidman, M. Reduction of shock frequency as reinforcement for avoidance. Journal of the Experimental Analysis of Behavior, 1962, 5, 247-257.

WEISMAN, A. Non-discriminated avoidance behavior in a large sample of rats. Psychological Reports, 1962, 10, 591-600. 